\title{
Glutamate Excitotoxicity in the Cerebellum Mediated by
} IL-1 $\beta$

\author{
Alma Nazlie Mohebiany ${ }^{1 \star}$ and Raphael Schneider ${ }^{2 \star}$ \\ ${ }^{1}$ Institute for Molecular Medicine, University Medical Center of the Johannes-Gutenberg University of Mainz, 55131 Mainz, Germany, and ${ }^{2}$ Department of \\ Medicine, Division of Neurology, University of Toronto, Toronto, Ontario, Canada M5S 1A8 \\ Review of Mandolesi et al.
}

Multiple sclerosis (MS) is the prototypic inflammatory demyelinating disorder of the CNS. Symptoms of cerebellar dysfunction, such as tremors and ataxia, are relatively common in MS, but available treatment options are generally of limited value. Although many clinical manifestations of MS are thought to arise from demyelination of axons that travel through the midbrain, pons, medulla, and cerebellum, there is emerging evidence that inflammation-driven excitotoxic neurodegeneration contributes to MS symptoms. For example, CSF from MS patients increases spontaneous EPSC (sEPSC) frequency and glutamate-mediated neuronal swelling in vitro, in an interleukin (IL)- $1 \beta$ dependent manner (Rossi et al., 2012). In a recent paper published in The Journal of Neuroscience, Mandolesi and colleagues (2013) investigated cerebellar glutamatergic transmission in an animal model of MS, experimental autoimmune encephalomyelitis (EAE), and the mechanisms underlying the synaptic alteration caused by IL- $1 \beta$.

Received Sept. 13, 2013; revised 0ct. 15, 2013; accepted 0ct. 17, 2013.

A.N.M. is the recipient of an Early Stage Researcher fellowship under the Marie Curie Action Initial Training Network project NEUROKINE-Neurological disorders orchestrated by cytoKines (EU FP7-PEOPLE-2012-ITN, project no. 316722).

${ }^{*}$ A.N.M and R.S. contributed equally to this work.

Correspondence should be addressed to Alma Nazlie Mohebiany, Universitätsmedizin, der Johannes Gutenberg-Universität, Institut für Molekulare Medizin, Obere Zahlbacher Strasse 67, 55131 Mainz, Germany. E-mail:amohebia@uni-mainz.de.

DOI:10.1523/JNEUROSCI.3946-13.2013

Copyright $\odot 2013$ the authors $\quad 0270-6474 / 13 / 3318353-03 \$ 15.00 / 0$
Astrocytes influence the duration and strength of synaptic signals by clearing glutamate from the synaptic cleft; failure to clear glutamate from the synapse leads to excitotoxic neurodegeneration (Rothstein et al., 1996). This important regulatory role depends on astrocytic expression of the high-affinity glutamate transporters GLAST/EAAT1 and GLT1/EAAT2. In the cerebellum, GLAST/EAAT1 is primarily expressed by Bergmann glia (BG), which are responsible for most of the glutamate clearance from the synaptic clefts of climbing and parallel fibers. Mandolesi and colleagues (2013) found that in the cerebellum of EAE mice, GLAST expression was decreased in concert with astrogliosis, and these effects were associated with prolonged spontaneous glutamatergic transmission.

Confirming a role for IL- $1 \beta$, brief ( 10 min) incubation of cerebellar slices with this cytokine increased the number of synaptic events and prolonged the decay time of the sEPSCs compared with controls or incubation with TNF- $\alpha$. Furthermore, briefly blocking endogenous IL- $1 \beta$ signaling in EAE cerebellar slices with IL-1 receptor antagonist (IL-1ra) resulted in partial rescue: sEPSCs in EAE slices incubated with IL-1ra were reduced compared with untreated EAE slices.

Mandolesi et al. (2013) found that endogenous sources of IL- $1 \beta$ at the symptomatic phase of disease in EAE mice included microglia/macrophages in white matter and infiltrating CD3 + lympho- cytes at white matter lesions and in the molecular layer. In fact, incubation of naive cerebellar slices with IL- $1 \beta$-producing $\mathrm{CD} 3+$ lymphocytes from the spleens of mice at the symptomatic phase of EAE was sufficient to induce the synaptic defects observed in EAE mice. Confirming the extent to which IL- $1 \beta$ mediates abnormal synaptic transmission in EAE mice, in vivo administration of IL-1ra prevented the inflammatory and synaptic events associated with EAE: mice had milder symptoms than control mice and GLAST expression levels in BG were near normal.

Mori and colleagues (2013) also found a correlation between the presence of infiltrating CD3 + lymphocytes in EAE and synaptic plasticity alterations. CD3+ lymphocytes of EAE mice, which release IL- $1 \beta$, altered synaptic plasticity in the hippocampi of these mice by promoting long-term potentiation (LTP) and potentially lowering the threshold of LTP induction by suppressing GABAergic transmission. These authors further observed that the diminished efficacy of GABAergic transmission mediated by EAE lymphocytes was prevented by coincubation with an IL- $1 \beta$ receptor antagonist. In addition, Nistico and colleagues (2013) showed that IL- $1 \beta$ secreted by activated microglia cells contributes to degeneration of GABAergic interneurons in the hippocampus of EAE mice, thus influencing synaptic function. Together, these results indicate that IL- $1 \beta$ produced by 
CD3 + lymphocytes or activated microglia alters synaptic transmission in EAE not only by enhancing glutamatergic transmission, but also by disrupting the balance between GABAergic and glutamatergic transmission.

Beyond altering synaptic transmission, glutamate excitotoxicity contributes to the lethal effect of IL- $1 \beta$ on oligodendrocytes, which is also relevant to MS pathology. In vitro experiments have shown that IL- $1 \beta$ is not directly cytotoxic to pure cultures of oligodendrocytes, but the presence of IL- $1 \beta$ along with microglia and astrocytes in coculture causes oligodendrocyte death (Takahashi et al., 2003). Antagonists of AMPA/kainate glutamate receptors blocked this IL- $1 \beta$-induced toxicity toward oligodendrocytes, providing a direct link between glia-mediated inflammation and tissue atrophy.

The effect of IL- $1 \beta$ on MS-like pathology is not limited to the EAE model of MS alone. Numerous investigations have extended our knowledge of MS pathology using other animal models in which pathology is induced using viruses or cuprizone. Theiler's murine encephalomyelitis virus (TMEV) establishes a persistent CNS infection in susceptible mouse strains that results in the development of chronic demyelinating disease. Cells infected with TMEV produce various cytokines, including IL-1 $\beta$. High levels of IL- $1 \beta$ were shown to promote demyelination in the TMEV model through induction of pathogenic Th17 cells (Sutton et al., 2006). In contrast, the presence of IL- $1 \beta$ produced by microglia and/or astrocytes has been shown to promote remyelination in a cuprizoneinduced animal model of multiple sclerosis (Mason et al., 2001).

Here, Mandolesi et al. (2013) identified infiltrating CD3 + lymphocytes as a primary source of IL- $1 \beta$ in the cerebellum through immunohistochemistry. Although most infiltrating CD3 + cells in EAE are CD $4+\mathrm{T}$ cells, there are multiple subsets of these cells, each with a unique cytokine profile, which will also influence the local cytokine milieu and thus the ongoing pathology. IL- $1 \beta$-activated astrocytes can secrete cytokines such as IL-6, GM-CSF, and TNF- $\alpha$, which influence T cell differentiation; other cytokines such as interferon-gamma or IL-17, released by activated $\mathrm{T}$ cell subsets, may also play an important role in this process by modifying or adding to the pro-inflammatory milieu and activation state of surrounding cells, not just astrocytes, thus contributing to glutamate excitotoxicity. For instance, a recent study showed that activated microglia release glutamate via hemichannels, resulting in increased glutamate uptake by astrocytes that subsequently respond by downregulating GLAST expression (Takaki et al., 2012). Therefore, it will be important to determine whether the IL- $1 \beta$-mediated glutamatergic excitotoxicity seen in the study by Mandolesi et al. (2013) resulted from immediate downstream effects of IL- $1 \beta$ receptor activation on astrocytes, other cytokines produced as a result of downstream signaling, or even a side-effect of microglial activation and glutamate production.

Defining specific aspects of the downstream effects of IL- $1 \beta$ is also important because this cytokine can have positive effects, and neuroinflammation may be required to ameliorate CNS diseases. Elevated levels of IL-1 are found in the brains of Alzheimer's disease (AD) patients, and has been thought to contribute to the pathology (Mrak and Griffin, 2000). However, a more recent study demonstrates that overexpression of IL- $1 \beta$ in the hippocampus of the APPswe/ PS1dE9 mouse model of AD significantly reduced levels of insoluble $\beta$-amyloid (Shaftel et al., 2007). In this APPswe/PS1dE9 AD mouse model of chronic neuroinflammation, IL- $1 \beta$ overexpression drove activation of microglia resulting in increased plaque phagocytosis. This beneficial neuroinflammatory response may serve as a homeostatic mechanism to counterbalance CNS injury. Indeed, in a recent report, delayed removal of myelin debris by microglia in the cuprizone model of MS resulted in impaired remyelination and oligodendrocyte precursor proliferation (Skripuletz et al., 2013). And so, while activated microglia found in CNS lesions in EAE and MS are generally thought to propagate inflammatory reactions, it is conceivable that enhanced clearance of myelin debris initiated by IL- $1 \beta$ may actually contribute to dampening inflammatory reactions in EAE and MS and allow for repair.

This study by Mandolesi and colleagues (2013) has potential implications not only for the treatment of MS, but also for other neurological diseases in which IL-1 $\beta$ may play a role, such as amyotrophic lateral sclerosis (ALS). While the pathogenicity of ALS is not completely understood, excitotoxicity has been implicated in the disease because of the efficacy of riluzole, a generic antiglutamatergic drug, in protecting motor neurons in animals (Bellingham, 2013). In ALS patients, riluzole has shown to slow disease progression and increase survival (Miller et al., 2012). Al- though often considered a consequence to neuronal injury and degeneration, inflammation is now recognized to be a prominent feature of ALS. In a recent study using a mouse model of ALS, deficiency in IL- $1 \beta$ or treatment with IL-1ra extended lifespan (Meissner et al., 2010). Thus, $\mathrm{IL}-1 \beta$-mediated glutamate excitotoxicity may be relevant for both MS and ALS. In conclusion, while the direct effects of IL- $1 \beta$ signaling will need to be further investigated, targeting more specific molecules and mechanisms involved in glutamatergic excitotoxicity in diseases such as MS and ALS may become an important avenue for the development of therapeutics.

\section{References}

Bellingham MC (2013) Pre- and postsynaptic mechanisms underlying inhibition of hypoglossal motor neuron excitability by riluzole. J Neurophysiol 110:1047-1061. CrossRef Medline

Mandolesi G, Musella A, Gentile A, Grasselli G, Haji N, Sepman H, Fresegna D, Bullitta S, De Vito F, Musumeci G, Di Sanza C, Strata P, Centonze D (2013) Interleukin-1beta alters glutamate transmission at Purkinje cell synapses in a mouse model of multiple sclerosis. J Neurosci 33:12105-12121. CrossRef Medline

Mason JL, Suzuki K, Chaplin DD, Matsushima GK (2001) Interleukin-1beta promotes repair of the CNS. J Neurosci 21:7046-7052. Medline

Meissner F, Molawi K, Zychlinsky A (2010) Mutant superoxide dismutase 1 -induced IL1 beta accelerates ALS pathogenesis. Proc Natl Acad Sci U S A 107:13046-13050. CrossRef Medline

Miller RG, Mitchell JD, Moore DH (2012) Riluzole for amyotrophic lateral sclerosis (ALS)/ motor neuron disease (MND). Cochrane Database Syst Rev 3:CD001447. CrossRef Medline

Mori F, Nistico R, Mandolesi G, Piccinin S, Mango D, Kusayanagi H, Berretta N, Bergami A, Gentile A, Musella A, Nicoletti CG, Nicoletti F, Buttari F, Mercuri NB, Martino G, Furlan R, Centonze D (2013) Interleukin-1beta promotes long-term potentiation in patients with multiple sclerosis. Neuromolecular Med. Advance online publication. doi:10.1007/ s12017-013-8249-7. CrossRef Medline

Mrak RE, Griffin WS (2000) Interleukin-1 and the immunogenetics of Alzheimer disease. J Neuropathol Exp Neurol 59:471-476. Medline

Nistico R, Mango D, Mandolesi G, Piccinin S, Berretta N, Pignatelli M, Feligioni M, Musella A, Gentile A, Mori F, Bernardi G, Nicoletti F, Mercuri NB, Centonze D (2013) Inflammation subverts hippocampal synaptic plasticity in experimental multiple sclerosis. PLoS One 8:e54666. CrossRef Medline

Rossi S, Furlan R, De Chiara V, Motta C, Studer V, Mori F, Musella A, Bergami A, Muzio L, Bernardi G, Battistini L, Martino G, Centonze D (2012) Interleukin-1beta causes synaptic hy- 
perexcitability in multiple sclerosis. Ann Neurol 71:76-83. CrossRef Medline

Rothstein JD, Dykes-Hoberg M, Pardo CA, Bristol LA, Jin L, Kuncl RW, Kanai Y, Hediger MA, Wang Y, Schielke JP, Welty DF (1996) Knockout of glutamate transporters reveals a major role for astroglial transport in excitotoxicity and clearance of glutamate. Neuron 16:675-686. CrossRef Medline

Shaftel SS, Kyrkanides S, Olschowka JA, Miller JN, Johnson RE, O’Banion MK (2007) Sustained hippocampal IL-1 beta overexpression mediates chronic neuroinflammation and ameliorates Alzheimer plaque pathology. J Clin Invest 117:1595-1604. CrossRef Medline

Skripuletz T, Hackstette D, Bauer K, Gudi V, Pul R, Voss E, Berger K, Kipp M, Baumgärtner W, Stangel M (2013) Astrocytes regulate myelin clearance through recruitment of microglia during cuprizone-induced demyelination. Brain 136:147-167. CrossRef Medline

Sutton C, Brereton C, Keogh B, Mills KH, Lavelle EC (2006) A crucial role for interleukin (IL)-1 in the induction of IL-17-producing T cells that mediate autoimmune encephalomyelitis. J Exp Med 203:1685-1691. CrossRef Medline
Takahashi JL, Giuliani F, Power C, Imai Y, Yong VW (2003) Interleukin-1beta promotes oligodendrocyte death through glutamate excitotoxicity. Ann Neurol 53:588-595. CrossRef Medline

Takaki J, Fujimori K, Miura M, Suzuki T, Sekino Y, Sato K (2012) L-glutamate released from activated microglia downregulates astrocytic L-glutamate transporter expression in neuroinflammation: the 'collusion' hypothesis for increased extracellular L-glutamate concentration in neuroinflammation. J Neuroinflammation 9:275. CrossRef Medline 